\title{
ANÁLISE DO BALANÇO HÍDRICO CLIMATOLÓGICO DECENDIAL DO PARQUE ESTADUAL DO RIO DOCE-PERD E ENTORNO: CLIMATOLOGIA DE 2005-2015 COMPARADA COM O ANO ANÔMALO 2013-2014
}

\author{
ANTUNES,Denise Aparecida - daa21@hotmail.com \\ Instituto Federal de Minas Gerais / IFMG \\ CUPOLILLO, Fulvio- fulvio.cupolillo@ifmg.edu.br \\ Instituto Federal de Minas Gerais / IFMG
}

\begin{abstract}
RESUMO: A região Sudeste do Brasil possui uma grande diversidade climática, devido a sua posição geográfica, e aos múltiplos mecanismos atmosféricos que atuam em todo o país. Esses fatores causam transformação da paisagem vegetal, na infiltração de água no solo, e desta forma provocam alterações a reposição hídrica de inúmeros sistemas ecológicos. Atentando para essas informações procurou-se realizar uma analogia dos balanços hídricos climatológicos decendiais da climatologia de 2005-2015, com o ano anômalo de 2013-2014. A área de estudo concentra-se no Parque Estadual do Rio DocePERD, um dos últimos fragmentos de Mata Atlântica no país. Neste trabalho utilizaram-se dados de quatro estações meteorológicas automáticas, duas pertencentes ao Instituto Nacional de Meteorologia - INMET, localizadas nos municípios de Timóteo e Caratinga, e duas pertencentes ao Instituto Mineiro de Gestão das Águas - IGAM, localizadas nos municípios de Ipatinga e em Marliéria no PERD. A metodologia aplicada baseou-se no Balanço Hídrico Climatológico decendial aplicado por diversos autores para o estudo da climatologia, a fim de detectar os possíveis mecanismos atmosféricos como: veranicos climatológicos, intensidade de deficiências e excedentes hídricos, ocorridos em decêndios não previstos pela climatologia. Espera-se que este trabalho contribua para a instalação de novos equipamentos automáticos, como uma rede de estações meteorológicas e pluviômetros, de modo que particularidades sejam descobertas, nos 42 lagos tectônicos dentro do bioma Mata Atlântica, do PERD.
\end{abstract}

PALAVRAS-CHAVE: balanço hídrico, deficiência, excedente, veranico.

ANALYSIS OF THE WATER BALANCE CLIMATOLOGICAL DECENDIAL OF THE STATE PARK OF RIO DOCE-PERD AND SURROUNDINGS: CLIMATOLOGY OF 2005-2015 COMPARED WITH THE YEAR ANOMALOUS 2013-2014

\begin{abstract}
The Southeastern region of Brazil has a great climatic diversity, due to its geographical position, and to the multiple atmospheric mechanisms that operate throughout the country. These factors cause transformation of the vegetation landscape, in the infiltration of water in the soil, and in this way cause changes in the water replenishment of numerous ecological systems. Attention to this information was sought to make an analogy of the decendial climatological water balance of the climatology of 2005-2015, with the anomalous year of 2013-2014. The study area is concentrated in the State Park of Rio Doce-PERD, one of the last fragments of Atlantic Forest in the country. Data from four automatic meteorological stations, two belonging to the National Institute of Meteorology - INMET, located in the municipalities of Timóteo and Caratinga, and two belonging to the Instituto Mineiro de Gestão das Águas - IGAM, located in the municipalities of Ipatinga and Marliéria in PERD. The applied methodology was based on the Decendial Climatological Water Balance applied by several authors for the study of climatology, in order to detect the possible atmospheric mechanisms such as: climatological summer, intensity of deficiencies and water surpluses, occurring in decays not predicted by climatology. It is hoped that this work will contribute to the installation of new automatic equipment, such as a network of meteorological stations and rain gauges, so that particularities are discovered in the 42 tectonic lakes within the Atlantic Forest biome of PERD.
\end{abstract}

KEYWORDS: Fire; Climate; Susceptibility; FWI.

\section{INTRODUÇÃO}

Ano 14 - Edição Especial Dossiê Climatologia de Minas Gerais - NOV 2018 
Para entender a dinâmica do clima é importante analisar um conjunto de fenômenos que se relacionam no tempo e no espaço, e criam unidades possíveis e serem medidas através do seu tamanho (escalas espaciais) e duração (escalas temporais). Os fenômenos climáticos são constituídos por um conjunto de elementos da natureza e dividem o mesmo tempo e espaço através de regimes de trocas energéticas e interdependentes. São três níveis estudados para entender o clima na Terra: a) nível zonal capaz de medir o clima em grandes extensões como mapas e cartas sinóticas; b) nível regional: determinam fatores como o relevo, que influenciam a circulação atmosférica na região; c) nível subregional: fatores como a geomorfologia, relevo e vegetação são analisados para determinar o clima; d) nível local: definido basicamente pela ação antrópica, o principal objetivo desse nível é produzir mapas de clima urbano.

Além das análises temporais e espaciais para identificar fenômenos atmosféricos, há na atmosfera porções de ar que se deslocam verticalmente e horizontalmente de acordo com mecanismos físicos que dão origens a anticiclones. Franca (2009) ao estudar tais porções observou que o aquecimento do planeta pelo sol produz movimentos verticais na atmosfera, que nas regiões quentes se expandem, e em regiões frias, o ar sofre contração e subsidência em latitudes altas. Essas porções deram origem a seis células, três em cada hemisfério: célula de Hadley, célula de Ferrel e células polares. (Fig.1)

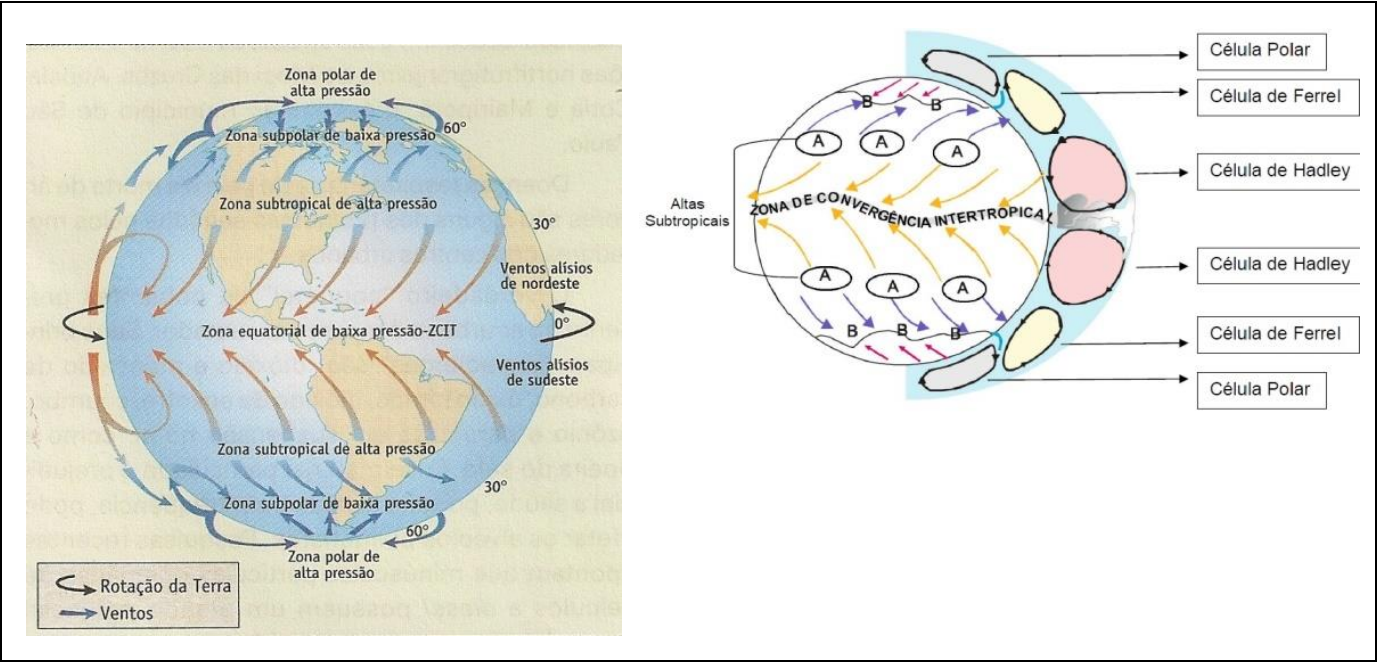

Figura 1 - Movimento geral da atmosfera (A), direção dos ventos;(B) localização das células de Hadley, Ferrel e Polar. Fonte: Instituto Geográfico de Agostine (1996); França 2009

Os movimentos de ar originados pelas células citadas anteriormente determinam variações nos padrões de circulação dos ventos originando sistemas meteorológicos (FIG.2). Um dos principais sistemas é denominado Zona de Convegência Intertropical -ZCIT é um importante sistema meteorológico atuante nos tropicos, de acordo com Carvalho e Oyama (2013) caracteriza-se pela presença de aglomerados convectivos, trata-se de um dos principais mecanismos de aquecimento da atmosferea ocasionando liberação de calor latente e interage com a radiação e nebulosidade. Para Molion e Bernardo (2002) trata-se de um dos principais sistemas que atuam na estação chuvosa do norte e nordeste. Os mesmos autores também definem outro importante sistema responsável pelas chuvas no Sudeste, o Anticiclone subtropical da América do Sul-ZCAS. Trata-se de uma faixa de nebulosidade persistente 
orientada de NW/SE, associada ao escoamento convergente na baixa troposfera do sul da Amazônia ao Atlântico Sul-Central percebida com maior intensidade no verão, esse sistema é um importante elemento na definição de situações atmosféricas no sudeste brasileiro, de acordo com Cavalcante et.al. (2009). Cupolillo (2015) define a ZCAS como, uma combinação de mecanismos atmosféricos atuantes no continente sul americano. Os mecanismos são: a Alta da Bolívia-AB, os Vórtices Ciclônicos de Alto Nível-VCAN, e os sistemas frontais vindo da porção meridional do continente. O ar úmido da superfície é transportado da parte norte para o sudeste do país. A ZCAS é formada por uma forte atividade convectiva e intensas precipitações denominada Alta da Bolívia$A B$, juntamente com sistemas frontais que formam uma esteira transportadora de umidade vindo do Pólos, ecausa intensas chuvas no Estado de Minas Gerais nos meses de dezembro e janeiro.
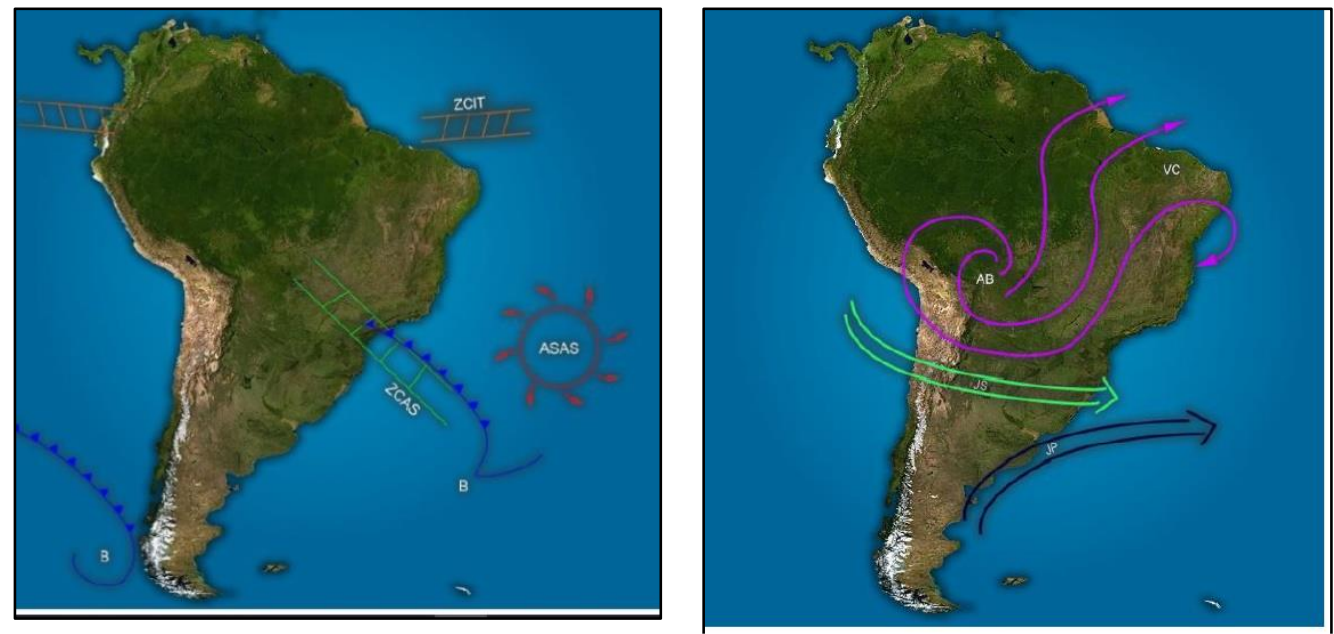

Figura 2 - Sistemas atmosféricos atuantes no Brasil e na região Sudeste.

Fonte: SOARES (2009).

A dinâmica climática do Estado de Minas Gerais proporciona uma ampla variabilidade, pois, encontra se em uma região tropical de transição climática. 0 estado possui duas estações distintas e bem definidas, um verão úmido e quente, que contrasta com um inverno seco e ameno, essa distinção acontece devido a diferentes sistemas atmosféricos atuantes no estado. Influente no Sudeste do país, e estudado por Soares (2009), o Anticiclone Subtropical do Atlântico Sul-ASAS é um sistema que exerce uma grande influência nas características de circulação atmosférica definindo bem sua atuação no verão, e no inverno. No verão, o ASAS age predominantemente sobre o oceano, facilitando forte influência de sistemas convectivos associados ao aquecimento continental, tal fato favorece a ocorrência de fortes precipitações nesse período.

No inverno, o deslocamento do anticiclone para o continente acaba induzindo o fenômeno de subsidência que caracteriza-se por céu sem nuvens, ausência de precipitações e favorece a poluição atmosférica. Para Bastos e Ferreira (2000), o ASAS (FIG.2) é de grande importância para o clima da América do Sul, no inverno ele inibe a entrada de frentes frias e causa a inversão térmica e a concentração de poluentes nos principais centros urbanos do sul e sudeste do país. Já no verão, o transporte de umidade nos baixos níveis da troposfera ao longo da ZCAS é afetado pelo ASAS, e faz com que a umidade 
se dirija para a região nordeste do Brasil. Outro fator dinâmico atmosférico é denominado Cavado do Nordeste-CN. Ele altera o regime de chuvas no Sudeste, por apresentar um escoamento de leste para oeste. Esse fator é classificado como um tipo de cavado invertido atuante em Minas Gerais, ocasionando longos períodos de estiagem na estação seca e veranicos na estação chuvosa, em função da forte subsidência do ar.

Esses momentos de estiagem são notados em distintas literaturas. Um exemplo são os veranicos, que Cupolillo (2015) define em seus estudos, e geram uma interrupção nas precipitações e provoca vários dias com baixos totais de precipitação, ou a ausência total de chuvas no período chuvoso. Em determinados locais o intervalo pode ser de 10 a 20 dias sem precipitação, podendo comprometer o abastecimento de água em regiões urbanas, ocasionando prejuízos para a agricultura e podendo interferir no desenvolvimento de biomas.

Há métodos para calcular se tais fenômenos atmosféricos interferem na vegetação de uma área. Um desses métodos é denominado Balanço Hídrico (BH). O BH, na definição de Tubelis, Nascimento (1986), diz respeito a uma técnica que contabiliza a quantidade de água disponível para as comunidades vegetais, levando em consideração a precipitação perante evapotranspiração potencial, com a capacidade de armazenamento de água no solo. (FIG.3). A disponibilidade de água nos solos está relacionada diretamente com o fator de concentração de vegetação mais do que com a precipitação, pois em uma área vegetada a infiltração é mais eficiente.

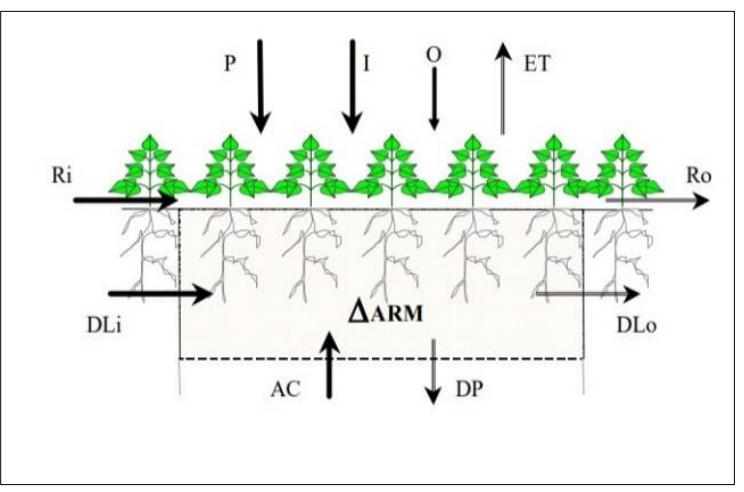
Entradas:
P: Precipitação
I: Irrigação
O: Orvalho
Ri: escoamento superficial
DLi: Drenagem lateral
AC: Ascensão capilar
Saídas:
ET: Evapotranspiração
Ro: Escoamento superficial
DLo: Drenagem lateral
DP: Drenagem profunda

Figura 3 - Esquema de entrada e saída de água no solo. Fonte: TUBELIS, NASCIMENTO (1986), Adaptação do autor.

O objetivo deste trabalho é analisar os balanços hídricos climatológicos decendiais pelo método de Thornthwaite e Matter (1955) para a climatologia dos anos de 2005a2015 comparando-a com o período anômalo de 2013e2014, no Parque Estadual do Rio Doce-PERD e seu entorno, com intuito de detectar o início das estações secas e chuvosas, a presença de veranicos climatológicos, possíveis anomalias na distribuição de chuvas, e identificar os mecanismos atmosféricos que atuam na superfície do PERD.

O PERD, (FIG.4), está localizado no trecho médio da bacia do rio Doce,

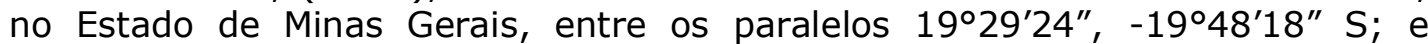
meridianos: $42^{\circ} 28^{\prime} 18^{\prime \prime},-42^{\circ} 38^{\prime} \mathrm{W}$, com limites geográficos a leste pelo Rio Doce e ao norte pelo Rio Piracicaba, possuindo 35.973ha, compreendem os 
municípios como Timóteo (14,1\%), Marliéria $(83,3 \%)$ e, Dionísio $(2,6 \%)$ na parte centro oeste (RELATORIO PELD-site 4, 2002). O PERD apresenta um dos últimos fragmentos de Mata Atlântica de Minas Gerais, que está sob administração do Instituto Estadual de Floresta-IEF desde 1962. O Parque é uma respeitável Unidade de Conservação-UC, que concentra importantes pesquisas em escala local, regional e nacional. Um dos projetos mais importantes é o Programa Brasileiro de Pesquisas de Longa Duração-PELD, de acordo com Peixoto (2012) é uma iniciativa da comunidade cientifica juntamente com o CNPq, com foco em pesquisa integrada constituída de uma rede de sites divididos pelos principais ecossistemas brasileiros, o sítio referente ao Parque Estadual do Rio Doce se encontra na página do PELD como o quarto sítio, Mata Atlântica e sistemas lacustres, e dispõe das mais variadas pesquisas e estudos da região do entorno do parque.

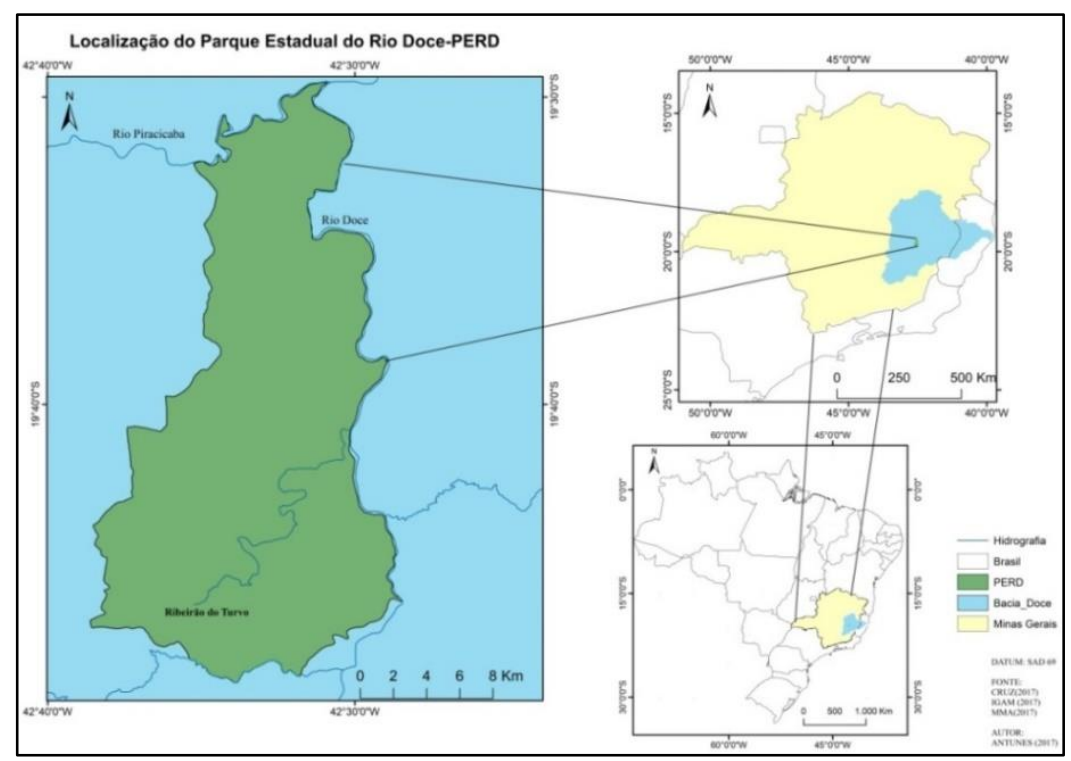

Figura 4 - Localização do PERD. Fonte: MMA, IGAM (2016)

\section{MATERIAL E MÉTODOS}

Para confeccionar os gráficos de balanços hídricos foram utilizados dados climatológicos de temperatura e chuvas das estações meteorológicas do Instituto Nacional de Meteorologia-INMET, e do Instituto Mineiro de Gestão das Aguas-IGAM, dos municípios de Ipatinga, Timóteo, Caratinga e MarliériaPERD,(FIG.5), TABELA 1, referente á 11 anos, períodos 2005 a 2015 e comparando-os com o ano anômalo de 2013 e 2014. 


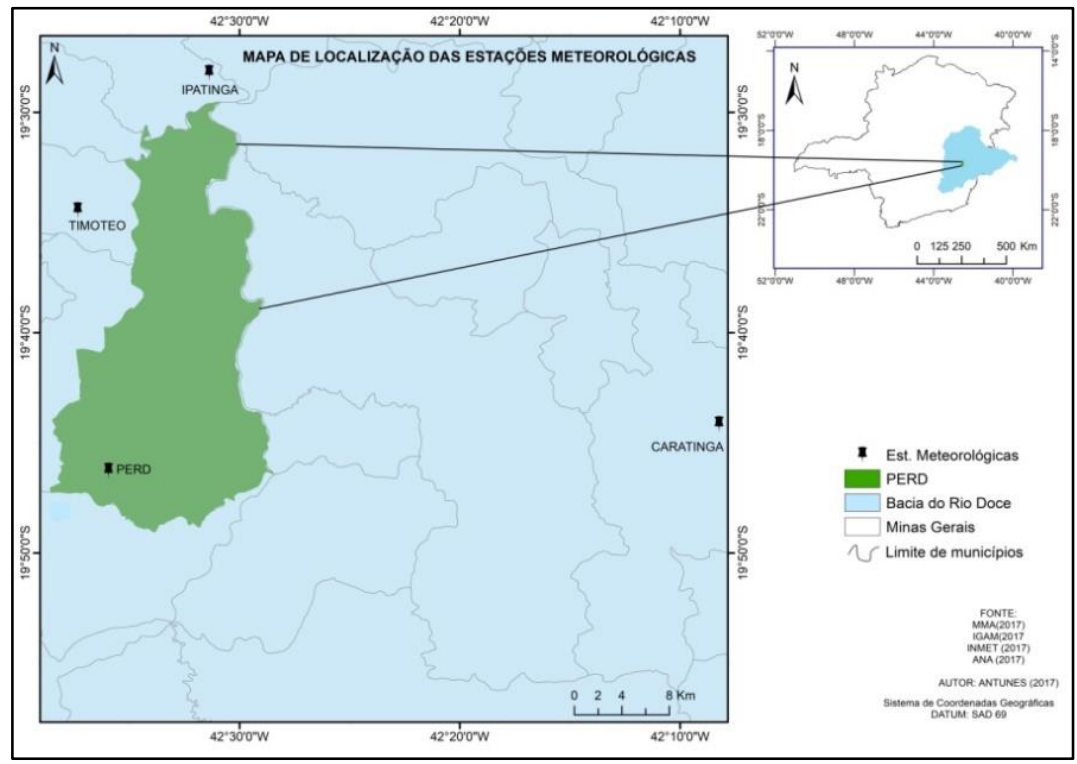

Figura 5 - Localização das estações meteorologicas do PERD e entorno. Fonte: INMET,

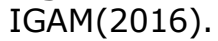

Tabela 1 - Identificação das estações meteorológicas

\begin{tabular}{lllll}
\hline LOCALIDADE & LAT & LONG & ALT(mts) & ID.EST.METEREOLOGICA \\
CARATINGA & 19,7357 & 42,13714 & 609 & AUT 554 \\
IPATINGA & 19,4700 & 42,52300 & 305 & 32507 \\
TIMOTEO & 19,5738 & 42,62241 & 493 & AUT 511 \\
PERD & 19,8030 & 42,63880 & 297 & 30800 \\
\hline
\end{tabular}

Fonte: INMET (2016), IGAM (2016).

Os dados foram dispostos no programa computacional Excel ${ }^{\mathrm{TM}}$ (ROLIM, 1998) (FIG.6) que calculou as médias das temperaturas e as somatórias de chuvas no período estudado. Segundo Nimer et.al (1989) a capacidade de campo adotada foi de $100 \mathrm{~mm}$. A representação do balanço hídrico facilita a visualização do ritmo anual de chuvas nas regiões analisadas. Os parâmetros para o calculo do balanço hídrico são : a) evapotranspiração-ETP; b)armazenamento de água no solo-ARM; c)Evapotranspiração Real-ETR; d)Precipitação-P; e)Deficiência hídrica-DEF; f) Excedente hídrico-EXC.

Os meses foram divididos por decêndios, períodos que equivalem dez dias consecutivos onde se calcula a média de uma variável. Então um mês corresponde a três decêndios e um ano trinta e seis decêndios, de acordo com Cupolillo (2015). Os decêndios foram distribuídos da seguinte maneira: nos meses com trinta e um dias a média do $3^{\circ}$ decêndio é elaborada com onze dias, o mês de fevereiro o $3^{\circ}$ decêndio é calculado para oito dias, e nos anos bissextos para nove dias. Para o cálculo dos períodos chuvosos e períodos secos utilizou equações desenvolvidas por Cupolillo (2015). 


\begin{tabular}{|c|c|c|c|c|c|c|c|c|c|c|c|c|c|}
\hline & attamento & $\begin{array}{r}\text { Glau } \\
\text { Pau } \\
\text { le Ciénc } \\
\text { DC }\end{array}$ & $\begin{array}{l}\text { e Sol } \\
\text { sar S } \\
\text { catas }\end{array}$ & & a e Meteorol & BHseg_V6.32002 & & & & & & & \\
\hline IDADE & & PER & & & LATITUD & $-19,80$ & & $\equiv$ & 110,7 & & & & \\
\hline EAD & 100 & & & & ANO & $2005-2015$ & & $a=$ & 2,46 & & & & \\
\hline e nåo for utilizar & esse parár & etro digita & (Igue & & NDA inicia & 1 & & & & & & & \\
\hline Irm do perioc & do anteri & & $=$ & & & & & & Tela & RL. - & & & \\
\hline Veg-Acum do & periodo & anterior & 0.00 & & Nümero c & le Linhas & 36 & Ajustar & & RLA) | & & & \\
\hline Tempo & Num de & NDA & $T$ & $\overline{\mathbf{P}}$ & $\mathbf{N}$ & ETP & P.ETP & NEG-AC & ARM & ALT & ETR & DEF & EXC \\
\hline DECENDIOS & Dias & & ${ }^{\circ} \mathrm{C}$ & $\mathrm{mm}$ & horas & Thornthwaite 1948 & $\mathrm{~mm}$ & & $\mathrm{~mm}$ & $\mathrm{~mm}$ & mm & $\mathrm{mm}$ & $\mathrm{mm}$ \\
\hline 01 & 10 & 1 & & & 13,2 & 0,00 & 0,0 & 0,0 & 100,00 & 0,00 & 0,0 & 0,0 & 0,0 \\
\hline 02 & 10 & 11 & & & 131 & 000 & 00 & 00 & 10000 & 0.00 & 00 & 00 & 00 \\
\hline 03 & 11 & 21 & & & 13,0 & 0,00 & 0,0 & 0,0 & 100,00 & 0,00 & 0,0 & 0,0 & 0,0 \\
\hline N1 & 10 & 32 & & & 12,9 & 0,00 & 0,0 & 0,0 & 100,00 & 0,00 & 0,0 & 0,0 & 0,0 \\
\hline N2 & 10 & 42 & & & 12,7 & 0,00 & 0,0 & 0,0 & 100,00 & 0,00 & 0,0 & 0,0 & 0,0 \\
\hline N3 & 8 & 52 & & & 12.5 & 0,00 & 0.0 & 0.0 & 100,00 & 0,00 & 0.0 & 0,0 & 0,0 \\
\hline D1 & 10 & 60 & & & 12,4 & 0,00 & 0,0 & 0,0 & 100,00 & 0,00 & 0.0 & 0,0 & 0.0 \\
\hline $\mathrm{D} 2$ & 10 & 70 & & & 12,2 & 0,00 & 0,0 & 0,0 & 100,00 & 0,00 & 0,0 & 0,0 & 0,0 \\
\hline D3 & 11 & 80 & & & 12,0 & 0,00 & 0.0 & 0.0 & 100,00 & 0,00 & 0.0 & 0.0 & 0.0 \\
\hline $\mathrm{J} 1$ & 10 & 91 & & & 11,8 & 0,00 & 0,0 & 0,0 & 100,00 & 0,00 & 0,0 & 0,0 & 0,0 \\
\hline $\mathrm{J} 2$ & 10 & 101 & & & 11,6 & 0,00 & 0,0 & 0,0 & 100,00 & 0,00 & 0,0 & 0,0 & 0,0 \\
\hline$\sqrt{3}$ & 10 & 111 & & & 11,4 & 0,00 & 0.0 & 0.0 & 100,00 & 0,00 & 0.0 & 0,0 & 0,0 \\
\hline $\mathrm{F} 1$ & 10 & 121 & & & 11,3 & 0.00 & 0.0 & 0.0 & 100,00 & 0,00 & 0.0 & 0.0 & 0,0 \\
\hline F2 & 10 & 131 & & & 11,1 & 0,00 & 0.0 & 0,0 & 100,00 & 0,00 & 0.0 & 0,0 & 0.0 \\
\hline$F_{3}$ & 11 & 141 & & & 11,0 & 0,00 & 0,0 & 0,0 & 100,00 & 0,00 & 0,0 & 0,0 & 0,0 \\
\hline M1 & 10 & 152 & & & 10,9 & 0,00 & 0.0 & 0,0 & 100,00 & 0,00 & 0,0 & 0,0 & 0,0 \\
\hline M2 & 10 & 162 & & & 108 & 0,00 & 0.0 & 0.0 & 100,00 & 0,00 & 0.0 & 0,0 & 0,0 \\
\hline$M_{3}$ & 10 & 172 & & & 10,8 & 0,00 & 0,0 & 0,0 & 100,00 & 0,00 & 0,0 & 0,0 & 0,0 \\
\hline A1 & 10 & 182 & & & 10,8 & 0,00 & 0.0 & 0.0 & 100,00 & 0,00 & 0.0 & 0.0 & 0,0 \\
\hline$A 2$ & 10 & 192 & & & 10.9 & 0,00 & 0.0 & 0.0 & 100,00 & 0,00 & 0.0 & 0.0 & 0,0 \\
\hline $\mathrm{A}_{3}$ & 11 & 202 & & & 11,0 & 0,00 & 0.0 & 0.0 & 100,00 & 0,00 & 0.0 & 0.0 & 0.0 \\
\hline M1 & 10 & 213 & & & 11,1 & 0,00 & 0,0 & 0,0 & 100,00 & 0,00 & 0,0 & 0,0 & 0,0 \\
\hline M2 & 10 & 223 & & & 11,3 & 0,00 & 0,0 & 0,0 & 100,00 & 0,00 & 0,0 & 0,0 & 0,0 \\
\hline M3 & 11 & 233 & & & 11.4 & 0.00 & 0.0 & 0.0 & 100,00 & 0,00 & 0.0 & 0.0 & 0.0 \\
\hline $\mathrm{J1}$ & 10 & 244 & & & 11,6 & 0,00 & 0,0 & 0,0 & 100,00 & 0,00 & 0,0 & 0,0 & 0,0 \\
\hline $\mathrm{J} 2$ & 10 & 254 & & & 11,8 & 0,00 & 0,0 & 0,0 & 100,00 & 0.00 & 0.0 & 00 & 0,0 \\
\hline$\sqrt{3} 3$ & 10 & 264 & & & 12,0 & 0,00 & 0,0 & 0,0 & 100,00 & 0,00 & 0,0 & 0,0 & 0,0 \\
\hline $\mathrm{J} 1$ & 10 & 274 & & & 12,2 & 0,00 & 0,0 & 0,0 & 100,00 & 0,00 & 0,0 & 0,0 & 0,0 \\
\hline $\mathrm{J} 2$ & 10 & 284 & & & 12,4 & 0,00 & 0,0 & 0,0 & 100,00 & 0,00 & 0,0 & 0,0 & 0,0 \\
\hline $\mathrm{J} 3$ & 11 & 294 & & & 12,6 & 0,00 & 0.0 & 0.0 & 100,00 & 0,00 & 0.0 & 0.0 & 0.0 \\
\hline A1 & 10 & 305 & & & 12,8 & 0,00 & 0,0 & 0,0 & 100,00 & 0,00 & 0,0 & 0,0 & 0,0 \\
\hline $\mathrm{A}_{2}$ & 10 & 315 & & & 12,9 & 0,00 & 0,0 & 0,0 & 100,00 & 0,00 & 0,0 & 0,0 & 0,0 \\
\hline $\mathrm{A} 3$ & 10 & 325 & & & 13,0 & 0,00 & 0,0 & 0.0 & 100,00 & 0.00 & 0.0 & 0.0 & 0,0 \\
\hline S1 & 10 & 335 & & & 13,1 & 0,00 & 0,0 & 0,0 & 100,00 & 0,00 & 0,0 & 0,0 & 0,0 \\
\hline S2 & 10 & 345 & & & 13.2 & 0,00 & 0,0 & 0,0 & 100,00 & 0,00 & 0,0 & 0,0 & 0,0 \\
\hline S3 3 & 11 & 355 & & & 13.2 & 0.00 & 0.0 & 0.0 & 100.00 & 0.00 & 0.0 & 0.0 & 0.0 \\
\hline TOTAIS & & & & & & & & & & & & & \\
\hline MÉDIAS & & & & & & & & & & & & & \\
\hline
\end{tabular}

Figura 6 - Programa excel adaptada por ROLIM (1998) para a realização do balanço hídrico climatológico. Fonte: ROLIM 1998

\section{RESULTADOS}

A estação meteorológica de Caratinga Figuras $7(a)$ e $7(b)$, mostram a distribuição de precipitação na climatologia, e no ano anômalo. Na climatologia a reposição hídrica inicia-se no $3^{\circ}$ decêndio de outubro, seguida por dez decêndios de excedente hídrico, e por um veranico no $3^{\circ}$ decêndio de fevereiro. Em março o excedente hídrico retorna estendendo-se até o $1^{\circ}$ decendio se abril. Quanto a estação seca, inicia-se no $2^{\circ}$ decendio de abril até o $2^{\circ}$ decendio de outubro.

No ano anômalo ocorre o excedente no $1^{\circ}$ decêndio de outubro, em seguida surge uma sequencia de seis veranicos intercalados, sendo o mais intenso o que ocorre do $3^{\circ}$ decêndio de janeiro até o $3^{\circ}$ decendio de fevereiro. Anomalamente, contradizendo a climatologia, o último veranico estende-se do $3^{\circ}$ decendio de abril até o $3^{\circ}$ decendio de maio.
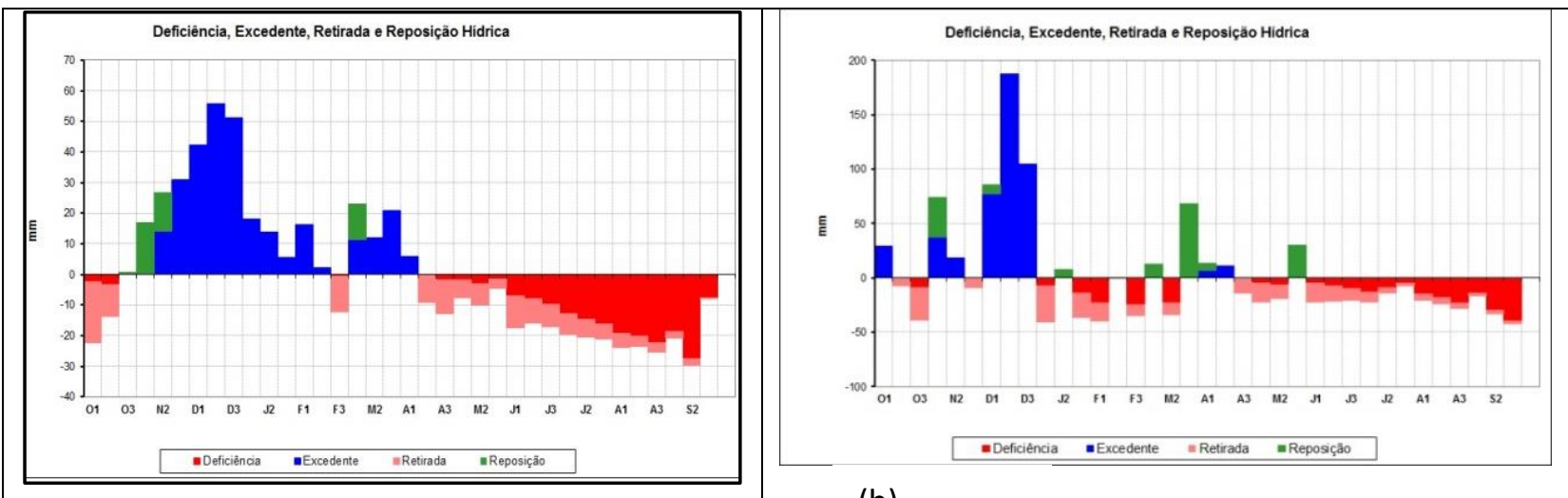

(b) 
(a)

Figura 7 - Balanços hídricos climatológicos decendiais no município de Caratinga; para os períodos 2005 a 2015 (a); e 2013-2014(b).

Tabela 2 - Resumo dos resultados dos decêndios na climatologia 2005 a 2015 , e ano anômalo 2013 e 2014 


\begin{tabular}{|c|c|c|c|c|c|}
\hline & \multicolumn{4}{|c|}{ CARATINGA 2005-2015 } & \\
\hline$\overline{D E C}$ & EXC & DEF & RET & REP & VERANICO \\
\hline $\mathrm{O} 1$ & & 8 & $\Delta$ & & \\
\hline $\mathrm{O} 2$ & & 0 & 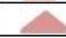 & & \\
\hline $\mathrm{O} 3$ & & & & $\square$ & \\
\hline $\mathrm{N} 1$ & & & & $\square$ & \\
\hline $\mathrm{N} 2$ & $>$ & & & $\square$ & \\
\hline $\mathrm{N} 3$ & $>$ & & & & \\
\hline D1 & 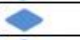 & & & & \\
\hline $\mathrm{D} 2$ & $>$ & & & & \\
\hline D3 & $>$ & & & & \\
\hline $\mathrm{J1}$ & 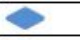 & & & & \\
\hline $\mathrm{J} 2$ & $>$ & & & & \\
\hline $\mathrm{J} 3$ & $>$ & & & & \\
\hline F1 & 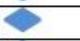 & & & & \\
\hline F2 & 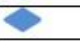 & & & & \\
\hline F3 & & & 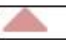 & & $\nabla$ \\
\hline M1 & $>$ & & & $\square$ & \\
\hline $\mathrm{M} 2$ & $>$ & & & & \\
\hline M3 & - & & & & \\
\hline $\mathrm{A} 1$ & 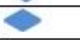 & & & & \\
\hline $\mathrm{A} 2$ & & & $\Delta$ & & \\
\hline $\mathrm{A} 3$ & & 0 & $\Delta$ & & \\
\hline M1 & & 0 & $\Delta$ & & \\
\hline $\mathrm{M} 2$ & & 0 & A & & \\
\hline M3 & & 0 & $\Delta$ & & \\
\hline $\mathrm{J} 1$ & & 0 & $\Delta$ & & \\
\hline $\mathrm{J} 2$ & & 0 & $\Delta$ & & \\
\hline $\mathrm{J} 3$ & & 0 & $\Delta$ & & \\
\hline $\mathrm{J} 1$ & & 0 & $\Delta$ & & \\
\hline $\mathrm{J} 2$ & & 0 & $\Delta$ & & \\
\hline $\mathrm{J} 3$ & & 0 & $\Delta$ & & \\
\hline A1 & & 8 & $\Delta$ & & \\
\hline $\mathrm{A} 2$ & & 0 & $\Delta$ & & \\
\hline $\mathrm{A}^{3}$ & & 0 & $\Delta$ & & \\
\hline S1 & & 0 & $\Delta$ & & \\
\hline S2 & & 8 & $\Delta$ & & \\
\hline S3 & & 0 & & & \\
\hline
\end{tabular}

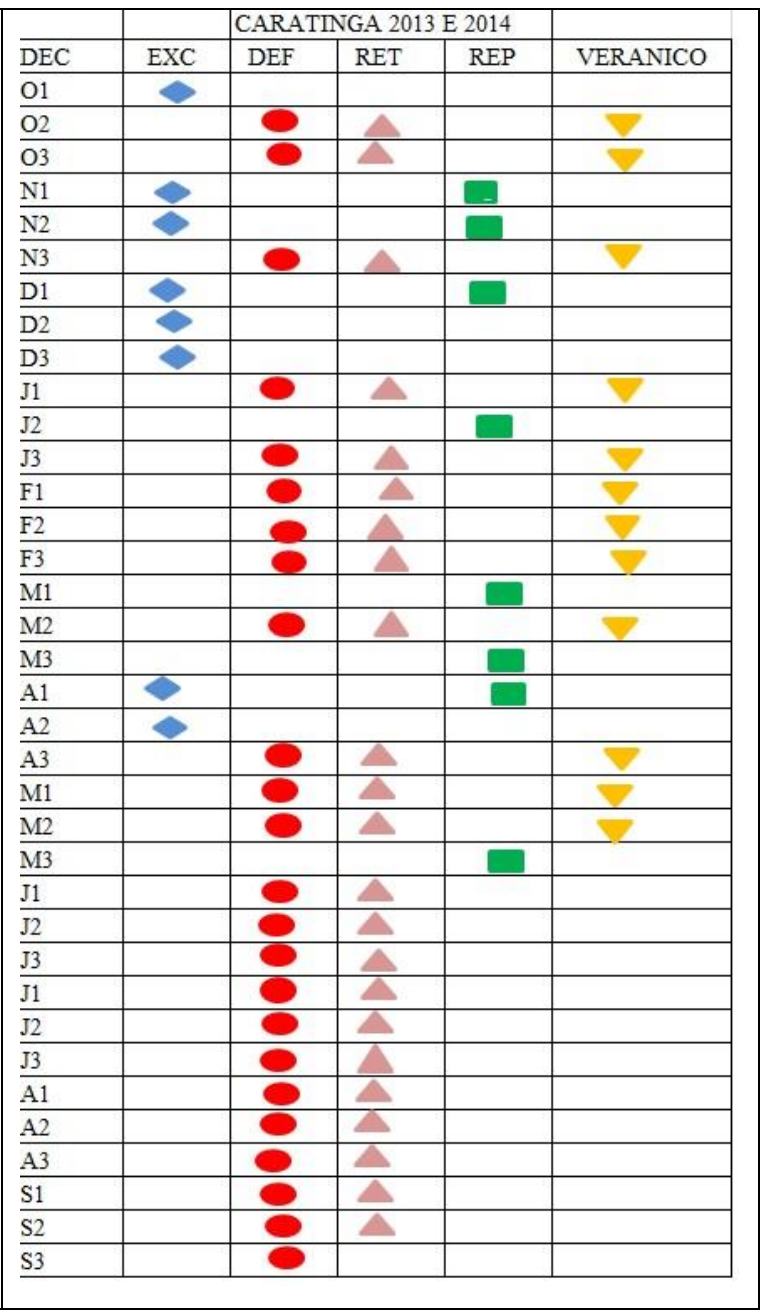

As Figuras 8 (a) e 8 (b), estação de Timóteo, apontam na climatologia o início da estação chuvosa a partir do $1^{\circ}$ decendio de novembro estendendo-se até $01^{\circ}$ decendio de abril. Neste período aparece a ocorrência de Veranico nos três decêndios de fevereiro. Quanto a estação seca, tem seu início no $2^{\circ}$ decendio de abril até o $3^{\circ}$ decendio de outubro.

No ano anômalo correspondente, observa-se o início da estação chuvosa no $1^{\circ}$ decendio de outubro finalizando no $3^{\circ}$ decendio de março. A anomalia deste ano está relacionada em uma sequencia de 6 veranicos intercalados. Apresentando como o mais intenso, no período do $3^{\circ}$ decendio de abril ao $3^{\circ}$ decendio de maio. Quanto a estação seca teve seu início no $1^{\circ}$ decendio de junho até o terceiro decendio de setembro, fugindo totalmente a climatologia apresentada. 


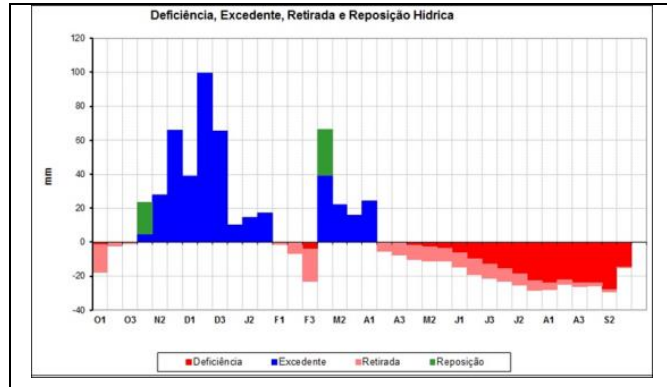

(a)

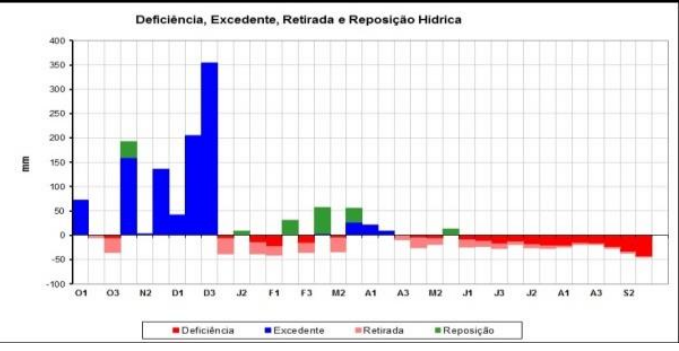

(b)

Figura 8 - Balanços hídricos climatológicos decendiais no município de Timóteo; para os períodos 2005 a 2015 (a); e 2013-2014(b).

Tabela 3 - Resumo dos resultados dos decêndios na climatologia 2005 a 2015 , e ano anômalo 2013 e 2014

\begin{tabular}{|c|c|c|c|c|c|c|c|c|c|c|c|}
\hline \multirow[b]{2}{*}{$(\mathrm{mm})$} & \multirow[b]{2}{*}{ EXC } & \multicolumn{3}{|c|}{ TIMOTEO 2005 a 2015} & \multirow[b]{2}{*}{ VERANICO } & \multirow[b]{2}{*}{$(\mathrm{mm})$} & \multicolumn{4}{|c|}{ TIMOTEO 2013 E 2014} & \multirow[b]{2}{*}{ VERANICC } \\
\hline & & DEF & RET & REP & & & EXC & DEF & RET & REP & \\
\hline 01 & & & $\Delta$ & & & $\mathrm{O} 1$ & $<$ & & & & \\
\hline $\mathrm{O} 2$ & & & & & & $\mathrm{O} 2$ & & & & & 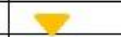 \\
\hline $\mathrm{O3}$ & & & $\Delta$ & & & $\overline{\mathrm{O} 3}$ & & & & & 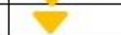 \\
\hline N1 & 8 & & & - & & $\mathrm{N} 1$ & $>$ & & & & \\
\hline N2 & $<$ & & & E & & $\mathrm{N} 2$ & $<$ & & & & \\
\hline N3 & $<$ & & & & & N3 & $<$ & & & & \\
\hline D1 & $<$ & & & & & D1 & $<$ & & & & \\
\hline D2 & $<$ & & & & & $\overline{\mathrm{D} 2}$ & $<$ & & & & \\
\hline D3 & 2 & & & & & D3 & $>$ & & & & \\
\hline $\mathrm{J} 1$ & 8 & & & & & $\mathrm{~J} 1$ & & & & & $\nabla$ \\
\hline $\mathrm{J} 2$ & $<$ & & & & & $\mathrm{J} 2$ & & & & & \\
\hline $\mathrm{J} 3$ & $<$ & & & & & $\mathrm{J3}$ & & & & & $\checkmark$ \\
\hline $\mathrm{F} 1$ & & & 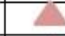 & & 8 & $\overline{\mathrm{F} 1}$ & & & & & $\nabla$ \\
\hline F2 & & & $\triangle$ & & 7 & $\mathrm{~F} 2$ & & & & & \\
\hline F3 & & & $\triangle$ & & 8 & F3 & & & & & 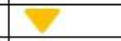 \\
\hline M1 & $\infty$ & & & & & $\overline{\mathrm{M} 1}$ & $<$ & & & & \\
\hline M2 & $<$ & & & & & $\mathrm{M} 2$ & & & & & 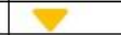 \\
\hline M3 & $<$ & & & & & M3 & $<$ & & & & \\
\hline A1 & $<$ & & & & & $\mathrm{A} 1$ & $<$ & & & & \\
\hline $\mathrm{A} 2$ & & & $Z$ & & & A2 & 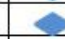 & & & & \\
\hline A3 & & & 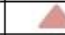 & & & A3 & & & & & 7 \\
\hline M1 & & & & & & M1 & & & & & 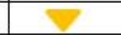 \\
\hline M2 & & & & & & $\mathrm{M} 2$ & & & & & 7 \\
\hline M3 & & & & & & M3 & $<$ & & & & \\
\hline $\mathrm{J} 1$ & & & & & & $\mathrm{J1}$ & & & & & \\
\hline $\mathrm{J} 2$ & & & 2 & & & $\mathrm{~J} 2$ & & & & & \\
\hline $\mathrm{J} 3$ & & & $\triangle$ & & & $\mathrm{J3}$ & & & & & \\
\hline $\mathrm{J} 1$ & & & & & & $\mathrm{~J} 1$ & & & & & \\
\hline $\mathrm{J} 2$ & & & $\angle$ & & & $\mathrm{J} 2$ & & & & & \\
\hline $\mathrm{J} 3$ & & & & & & $\mathrm{J3}$ & & & & & \\
\hline $\mathrm{A} 1$ & & & $\angle$ & & & A1 & & & & & \\
\hline $\mathrm{A} 2$ & & & & & & $\mathrm{~A} 2$ & & & & & \\
\hline A3 & & & & & & $\mathrm{A} 3$ & & & & & \\
\hline S1 & & & & & & $\mathrm{S1}$ & & & & & \\
\hline S2 & & & $A$ & & & $\mathrm{~S} 2$ & & & & & \\
\hline S3 & & & & & & $\overline{\mathrm{S} 3}$ & & & & & \\
\hline
\end{tabular}

No PERD, Figuras 9(a) e 9(b), a climatologia inicia a estação chuvosa no $3^{\circ}$ decêndio de outubro com reposição/excedente até $01^{\circ}$ decêndio de abril. Nota-se, duas sequencias de veranicos, uma com menor intensidade, ocorrendo 
no $1^{\circ}$ decendio de novembro, e a outra com maior intensidade, ocorrendo nos decêndios de fevereiro.

No ano anômalo a estação chuvosa inicia-se no $3^{\circ}$ decendio de outubro, finalizando-se no $2^{\circ}$ decendio de março. Neste período, surgem duas sequências de veranico. A primeira, com menor intensidade, no $1^{\circ}$ decendio de novembro e a segunda, com maior intensidade, nos decêndios de janeiro e $1^{\circ}$ e $2^{\circ}$ de fevereiro.

Quanto a estação seca, inicia-se no $3^{\circ}$ decendio de março estendendo-se até o $2^{\circ}$ decendio de outubro.

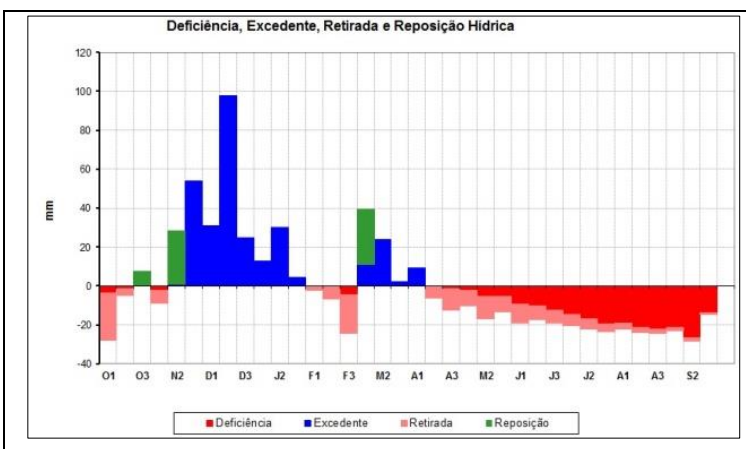

(a)

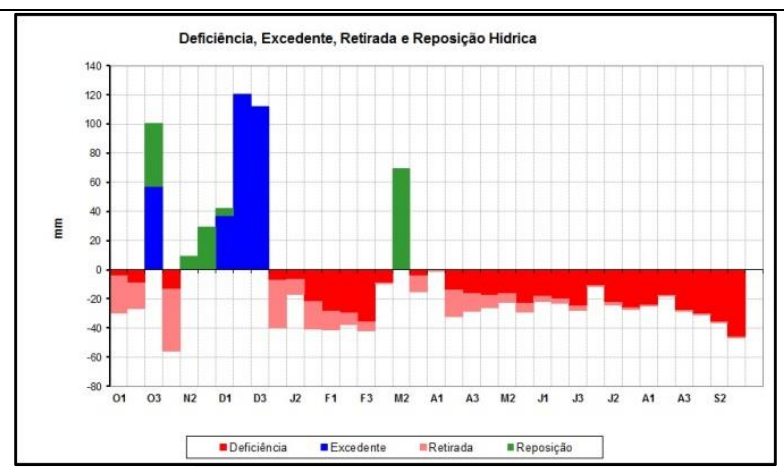

(b)

Figura 9 - Balanços hídricos climatológicos decendiais do PERD; para os períodos 2005 a 2015 (a); e 2013-2014(b). 
Tabela 3 - Resumo dos resultados dos decêndios na climatologia 2005 a 2015 , e ano anômalo 2013 e 2014.

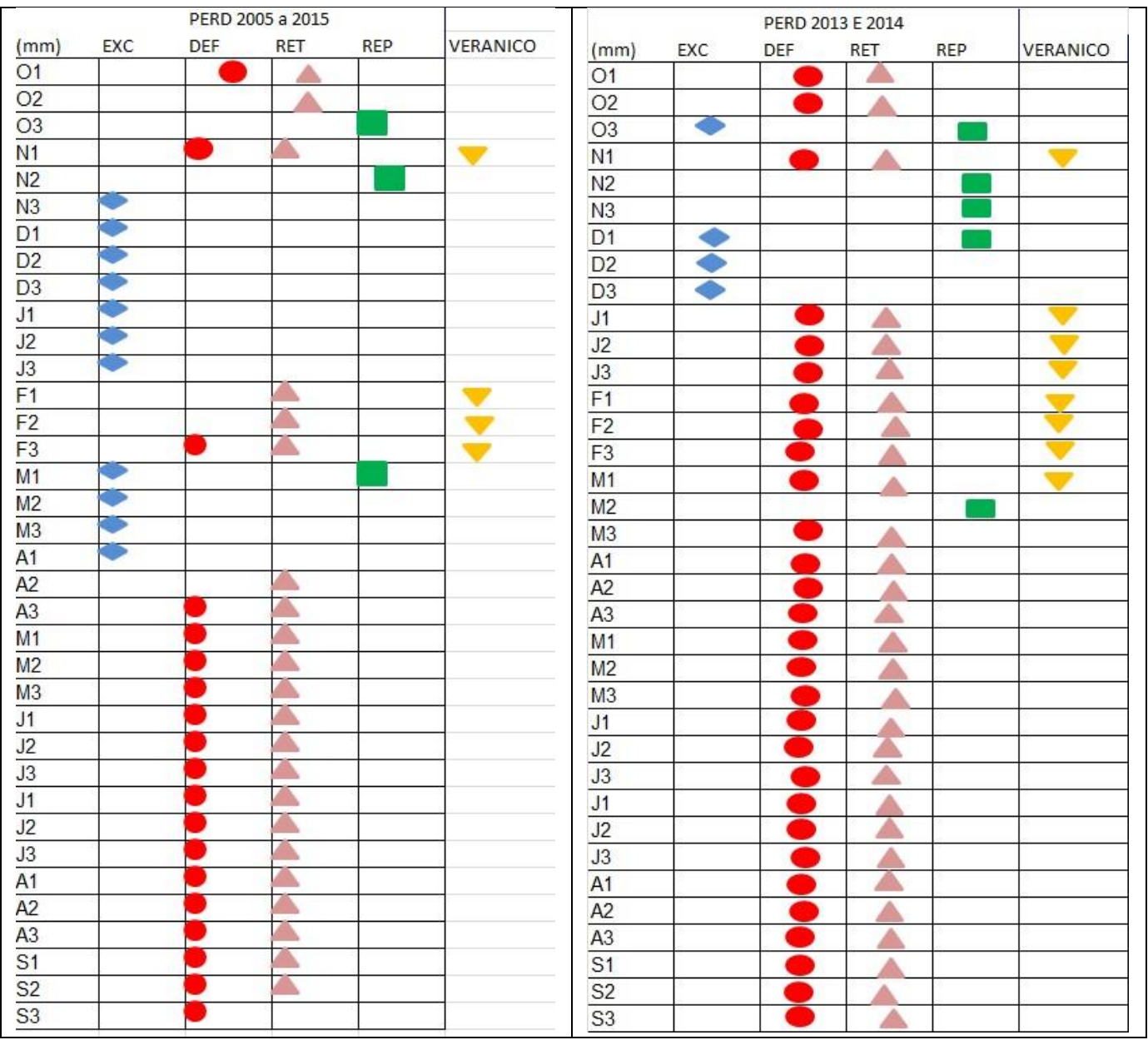

Em Ipatinga, Figuras 10 (a) e 10 (b), a climatologia, segue o mesmo padrão dos decêndios das outras localidades, com o início da estação chuvosa no $3^{\circ}$ decêndio de outubro, finalizando-se no $1^{\circ}$ decêndio de abril. Uma única sequencia de veranico, é encontrada nos decêndios de fevereiro. Quanto a estação seca inicia-se no $2^{\circ}$ decêndio de abril, finalizando-se no $2^{\circ}$ decendio de outubro.

No ano anômalo, a estação chuvosa inicia-se no $1^{\circ}$ decêndio de outubro tendo seu termino no $2^{\circ}$ decendio de maio. Apresenta 6 sequencias de veranicos, intercalados, sendo o de maior intensidade do $2^{\circ}$ decendio de abril ao $1^{\circ}$ decendio de maio.

No que se refere a estação seca, inicia-se no $3^{\circ}$ decendio de maio e tem seu término no $3^{\circ}$ decendio de setembro. 

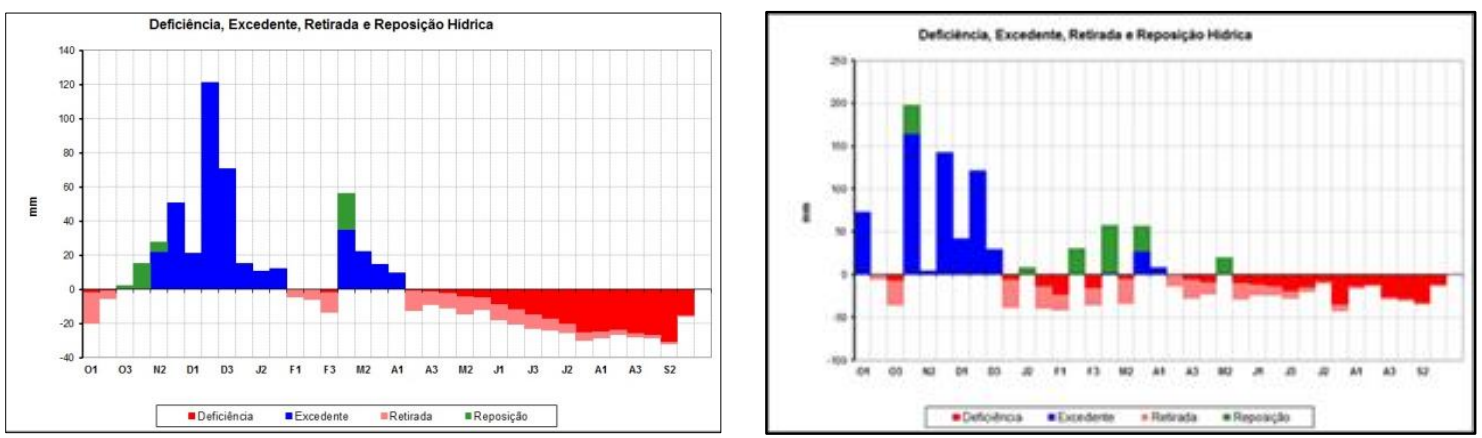

Figura 10 - Balanços hídricos climatológicos decendiais do município de Ipatinga; para os períodos 2005 a 2015 (a); e 2013-2014(b).

Tabela 4 - Resumo dos resultados dos decêndios na climatologia 2005 a 2015 , e ano anômalo 2013 e 2014.

\begin{tabular}{|c|c|c|c|c|c|c|c|c|c|c|c|}
\hline \multirow[b]{2}{*}{$(\mathrm{mm})$} & \multicolumn{4}{|c|}{ IPATINGA 2005 a 2015} & \multirow[b]{2}{*}{ VERANICO } & \multicolumn{4}{|c|}{ IPATINGA 2013 E 2014} & \multirow[b]{2}{*}{ REP } & \multirow[b]{2}{*}{ VERANICO } \\
\hline & EXC & DEF & RET & REP & & $\overline{\mathrm{DEC}}$ & EXC & DEF & RET & & \\
\hline 01 & & 0 & A & & & o1 & $<$ & & & & \\
\hline $\mathrm{O} 2$ & & & - & & & $\underline{\mathrm{O} 2}$ & & O & $\Delta$ & & $\nabla$ \\
\hline $\mathrm{O} 3$ & & & & E & & 03 & & 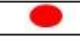 & $\Delta$ & & $\nabla$ \\
\hline $\mathrm{N1}$ & & & & E & & N1 & $>$ & & & a & \\
\hline N2 & 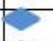 & & & L & & N2 & 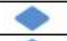 & & & $\square$ & \\
\hline N3 & 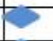 & & & & & N3 & 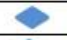 & & & & \\
\hline D1 & 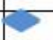 & & & & & D1 & $<$ & & & & \\
\hline D2 & 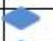 & & & & & $\overline{\mathrm{D} 2}$ & - & & & & \\
\hline D3 & 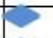 & & & & & D3 & 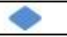 & & & & \\
\hline $\mathrm{J1}$ & 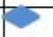 & & & & & $\mathrm{J1}$ & & 0 & $\Delta$ & & $\nabla$ \\
\hline $\mathrm{J} 2$ & 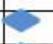 & & & & & $\mathrm{J} 2$ & & & & $\square$ & \\
\hline $\mathrm{J} 3$ & 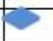 & & & & & $\mathrm{J} 3$ & & $?$ & $\Delta$ & & $\nabla$ \\
\hline F1 & & & to & & $\nabla$ & $\mathrm{F} 1$ & & 0 & $\Delta$ & & $z$ \\
\hline $\mathrm{F} 2$ & & & 5 & & $\nabla$ & $\mathrm{F} 2$ & & & & $\square$ & \\
\hline F3 & & 0 & to & & 8 & F3 & & 0 & $\Delta$ & & $\nabla$ \\
\hline M1 & 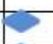 & & & & $\sqrt{2}$ & M1 & $>$ & & & $\square$ & \\
\hline$\overline{\mathrm{M} 2}$ & 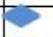 & & 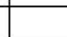 & & & $\mathrm{M} 2$ & & 0 & $\Delta$ & & $\nabla$ \\
\hline $\mathrm{M} 3$ & 0 & & 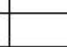 & & & M3 & $<$ & & & $\square$ & \\
\hline A1 & 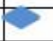 & & & & & $\mathrm{A} 1$ & $>$ & & & & \\
\hline$\overline{\mathrm{A} 2}$ & & & $t$ & & & $\mathrm{~A} 2$ & & 0 & $\Delta$ & & $\nabla$ \\
\hline$\overline{\mathrm{A} 3}$ & & & to & & & $\mathrm{A} 3$ & & 0 & $\Delta$ & & $\nabla$ \\
\hline$\overline{\mathrm{M} 1}$ & & & 5 & & & M1 & & 0 & & & $\nabla$ \\
\hline M2 & & 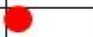 & $t$ & & & M2 & & & & L & \\
\hline $\bar{M} 3$ & & & $t$ & & & M3 & & 0 & $\Delta$ & & \\
\hline $\mathrm{J1}$ & & 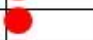 & 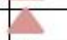 & & & $\mathrm{J} 1$ & & 0 & $\Delta$ & & \\
\hline $\mathrm{J} 2$ & & 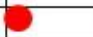 & $t$ & & & $\mathrm{~J} 2$ & & ? & $\Delta$ & & \\
\hline $\mathrm{J} 3$ & & 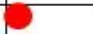 & 十 & & & $\mathrm{J} 3$ & & 0 & $\Delta$ & & \\
\hline $\mathrm{J} 1$ & & 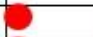 & t> & & & $\mathrm{J} 1$ & & 0 & $\Delta$ & & \\
\hline $\mathrm{J} 2$ & & 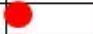 & t & & & $\frac{\mathrm{J} 2}{2}$ & & 0 & $\Delta$ & & \\
\hline $\mathrm{J} 3$ & & 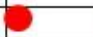 & 7 & & & $\mathrm{J3}$ & & 0 & $\Delta$ & & \\
\hline$\overline{\mathrm{A} 1}$ & & 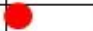 & 7 & & & $\mathrm{~A} 1$ & & 0 & $\Delta$ & & \\
\hline A2 & & 5 & 7 & & & $\mathrm{~A} 2$ & & 0 & $\Delta$ & & \\
\hline$\overline{\mathrm{A} 3}$ & & & t & & & $\mathrm{A} 3$ & & 0 & $\Delta$ & & \\
\hline S1 & & 3 & 5 & & & $\underline{S 1}$ & & 0 & $\Delta$ & & \\
\hline S2 & & $\pi$ & t & & & $\underline{\mathbf{S} 2}$ & & 0 & $\Delta$ & & \\
\hline & & & & & & $\underline{\mathrm{S} 3}$ & & 0 & $\Delta$ & & \\
\hline
\end{tabular}

\section{CONCLUSÃO}

Verificou-se, quanto a climatologia, que o início da estação chuvosa aparece no $3^{\circ}$ decêndio de outubro, e que coincide com a entrada dos sistemas 
frontais na região do PERD. Os mecanismos atmosféricos de mesoescala como a ZCAS, AB e o ASAS, assim como, o CN e o VCAN tem forte atuação na estação chuvosa. A ZCAS responsável pelas invernadas de verão levando a chuvas intensas. O ASAS juntamente com o CN e/ou VCAN, são responsáveis pela formação de veranicos climatológicos que normalmente ocorrem nos decêndios de fevereiro.

Para a climatologia de 2005 a 2015, a estação chuvosa estende-se de outubro a março, e a estação seca de abril a setembro.

A diversidade, nos anos anômalos, das sequencias de veranicos nas 4 localidades, sugere a influencia da rugosidade da superfície interagindo com os efeitos destes mecanismos atmosféricos.

Salienta-se a realização de futuros estudos para uma ampla discussão a cerca dos fenômenos atmosféricos que interferem no regime de chuvas no PERD. Para tal, necessário se faz em ampliar a rede de estações meteorológicas na região, ampliando dete modo o banco de dados meteorológicos.

\section{AGRADECIMENTOS}

Ao Instituto Federal de Minas Gerais -IFMG, Instituto Nacional de Meteorologia-INMET e ao Instituo Mineiro de Gestão das Águas - IGAM.

\section{REFERÊNCIAS}

BASTOS, C.; FERREIRA, N. Análise Climatológica da Alta Subtropical do

Atlântico Sul. In: CONGRESSO BRASILEIRO DE METEOROLOGIA,11., 2000.Rio de Janeiro. Anais... Rio de Janeiro, 2000. p. 612-619.

CARVALHO, M.A.V; OYAMA,M.D. Variabilidade da largura e intensidade da Zona de Convergência Intertropical Atlântico: Aspectos observacionais. Revista Brasileira de Meteorologia, v.28,n.3,305-316, 2013.

CAVALCANTI, I.F.A.; FERREIRA, N.J.; SILVA, M.G.A.J; DIAS,M.A.F. Tempo e Clima no Brasil. São Paulo: Oficina de textos, 2009.463p.

CUPOLILLO, F. Diagnóstico Hidroclimatológico da Bacia do Rio Doce. $1^{\text {a }}$. ed. Saarbrücken - Alemanha: Novas Edições Acadêmicas, 2015. v. 1. 224p

INSTITUTO ESTADUAL DE FLORESTA. Plano de Manejo do Parque Estadual do Rio Doce.2008387,1990.

MINAS GERAIS. Decreto-Lei $n^{\circ} 1.119$, de 14 de julho de 1944 . Dispõe sobre parques florestais. Diario executivo, Minas Gerais, 15 de julho 1944.

MOLION, L.C.B.; BERNARDO, S.O. Uma revisão da Dinâmica das chuvas no Nordeste brasileiro. Revista Brasileira de Meteorologia,v.17,n.1,p1-10,2002.

NIMER, Edmon. Climatologia do Brasil. 2a ed. Rio de Janeiro: Instituto Brasileiro de Geografia e Estatística - IBGE, 1989b. 422 p.

PEIXOTO,E.L. Caracterização e Perspectivas do Parque Estadual do Rio DoceMG: Uma abordagem a partir de imagens de sensoriamento remoto e fotografias hemisféricas de Dossel. 105p. Dissertação (mestrado em análise e modelagem 
de sistemas ambientais)- Instituto de Geociências, Universidade Federal de Minas Gerais - UFMG, Belo Horizonte, 2012.

PEREIRA, Antônio Roberto; ANGELOCCI, Luiz Roberto; SENTELHAS, Paulo César. Agrometeorologia: fundamentos e aplicações prática. Guaíba: Agropecuária, 2002. p.478 .

RELATÓRIO CIENTIFICO DAS ATIVIDADES DO PROGRAMA DE PESQUISAS ECOLÓGICAS DE LONGA DURAÇÃO, site 4, Mata Atlântica e Sistema Lacustre do Médio Rio Doce. Setembro de 2002. p.391.

ROLIM, Glauco de Souza. el al. Planilhas no ambiente EXCELTM para os cálculos de balanços hídricos: normal, sequencial, de cultura e de produtividade real e potencial. Revista Brasileira de Agrometeorologia, Santa Maria, v. 6, n.1, p 133137, 1998.

SOARES, H.C. Estudo das condições atmosféricas e oceânicas do Atlântico Sudoeste e suas associações com extremos de captura da sardinha-verdadeira. 2009.150f. Dissertação (mestrado em Meteorologia)- Instituto Nacional de Pesquisa Espaciais-INPE, São José dos Campos, 2009. Disponível em: http://mtc-m16c.sid.inpe.br/col/sid.inpe.br/mtc-

m18@80/2009/03.05.11.39/doc/publicacao.pdf. Acesso em: 06 nov.2017.

TUBELIS, Antônio; NASCIMENTO, Fernando José Lino do. Meteorologia descritiva: fundamentos e aplicações brasileiras. São Paulo; Nobel. 1 ed.4reimp.1986. p.374.

THORNTHWAITE, C. W.; MATHER, J. R. The water balance. Publications In Climatology. Drexel Institute of tecnology. New Jersey.v.8, n1.1955.p. 104. Disponível em: oregondigital.org/catalog/oregondigital:df70pr001\#page/13/mode/1up. Acesso em: 28 jun. 2017 